\title{
Climacteric Lowers Plasma Levels of Platelet-Derived Microparticles: A Pilot Study in Pre- versus Postmenopausal Women
}

\author{
Andreas Rank ${ }^{b} \quad$ Rienk Nieuwland $^{a} \quad$ Sabine Roesner ${ }^{c}$ Katharina Nikolajek ${ }^{d}$ \\ Erhard Hiller ${ }^{\mathrm{e}}$ Bettina Toth ${ }^{\mathrm{c}}$ \\ ${ }^{a}$ Department of Clinical Chemistry, Academic Medical Center, Amsterdam, The Netherlands; \\ b2. Medizinische Klinik, Klinikum Augsburg, Augsburg, 'Department of Gynecological Endocrinology and \\ Fertility Disorders, Ruprecht-Karls University, Heidelberg, ${ }^{\mathrm{d} D}$ Department of Radiotherapy, and \\ e Medizinische Klinik III, Campus Grosshadern, Klinikum der Ludwig Maximilians Universität München, \\ München, Germany
}

\section{Key Words}

Microparticles $\cdot$ Climacteric $\cdot$ Thrombosis $\cdot$ Platelets

\begin{abstract}
Background: Climacteric increases the risk of thrombotic events by alteration of plasmatic coagulation. Up to now, less is known about changes in platelet- (PMP) and endothelial cell-derived microparticles (EMP). Methods: In this prospective study, plasma levels of microparticles (MP) were compared in 21 premenopausal and 19 postmenopausal women. Results: No altered numbers of total MP or EMP were measured within the study groups. However, the plasma values of CD61-exposing MP from platelets/megakaryocytes were higher in premenopausal women $\left(5,364 \times 10^{6} / /\right.$, range $4,384-17,167)$ as compared to postmenopausal women $\left(3,808 \times 10^{6} / \mathrm{l}\right.$, range $\left.2,009-8,850 ; p=0.020\right)$. This differentiation was also significant for the subgroup of premenopausal women without hormonal contraceptives $(5,364 \times$ $10^{6} / \mathrm{l}$, range 4,223-15,916; $\left.p=0.047 ; n=15\right)$. Furthermore, in premenopausal women, higher plasma levels of PMP exposing CD62P were also present as compared to postmenopausal women $\left(288 \times 10^{6} / \mathrm{l}\right.$, range $139-462$, vs. $121 \times 10^{6} / \mathrm{l}$, range 74-284; $p=0.024)$. This difference was also true for CD63+ PMP levels $\left(281 \times 10^{6} / \mathrm{l}\right.$, range $182-551$, vs. $137 \times$
\end{abstract}

$10^{6} / \mathrm{l}$, range 64-432; $\left.\mathrm{p}=0.015\right)$. Conclusion: Climacteric lowers the level of PMP but has no impact on the number of EMP in women. These data suggest that PMP and EMP do not play a significant role in enhancing the risk of thrombotic events in healthy, postmenopausal women.

Copyright $\odot 2012$ S. Karger AG, Basel

\section{Introduction}

In premenopausal women with regular menstrual cycles, the hormonal axis is controlled by the hypothalamus and the hypophysis. Follicle-stimulating hormone (FSH) released in the hypophysis stimulates the maturation of ovarian follicles, which in turn produce estradiol. Exerting a positive feedback, the increasing estradiol concentrations lead to elevated levels of luteinizing hormone during the follicular phase of the menstrual cycle triggering ovulation. During treatment with oral contraceptives (OC), the continuous administration of gestagens or the combined administration of gestagens and ethinylestradiol results in constant hormonal levels under which FSH and luteinizing hormone concentrations remain unchanged [1].

\section{KARGER}

Fax +4161306 1234

E-Mail karger@karger.ch

www.karger.com
(C) 2012 S. Karger AG, Basel

0001-5792/12/1281-0053\$38.00/0

Accessible online at:

www.karger.com/aha
Dr. med. Andreas Rank

2. Medizinische Klinik, Klinikum Augsburg

Stenglinstrasse 2, DE-86156 Augsburg (Germany)

Tel. +49821 4002353

E-Mail andreas.rank@klinikum-augsburg.de 
In Europe, women reach the menopause at around the age of 54 years [2]. At this time, there are less than a thousand follicles remaining in the ovaries leading to decreased levels of estradiol [3]. Through a feedback mechanism, the hypophysis increases the production and distribution of FSH in order to stimulate the ovaries. Menopausal symptoms such as hot flushes, sweating, vaginal atrophy and osteoporosis are a consequence of the decreased estadiol levels. However, climacteric is also responsible for a dramatic increase in the risk of adverse thrombotic events such as myocardial infarction, stroke, deep venous thrombosis and pulmonary embolism [4].

Up to now, most investigations regarding the changes in the coagulation system focus on plasmatic factors and show inconstant laboratory findings: plasma concentrations of fibrinogen and fibrin $\mathrm{D}$-dimer, both markers of thrombotic risk, are elevated, and endogenous fibrinolytic activity is reduced in healthy postmenopausal women as compared to younger women [5]; however, inhibitors like protein $\mathrm{C}$, protein $\mathrm{S}$, antithrombin, or tissue factor pathway inhibitor are also increased in menopausal women [6]. In view of these findings, climacteric seems to destabilize the plasmatic coagulation system.

Less is known about changes of platelets and endothelial cells which could also play a role in the observed enhancement of thrombotic risk during aging. One way to determine the activation status of platelets as well as of endothelial cells in vivo is to measure microparticle (MP) concentrations in plasma. MP can be released from every blood and endothelial cell into the peripheral circulation. However, in healthy humans, MP are mainly CD61+ and are derived from megakaryocytes or from platelets (platelet-derived microparticles, PMP) with additional exposing of the platelet activation markers P-selectin and CD63 [7]. In particular, the composition and function of PMP have been intensively analyzed $[8,9]$. PMP are major players in coagulation [10] as they are highly thrombogenic [11] because of their phospholipid surfaces with binding sites for several different coagulation factors [12, 13]. PMP are important initiators of coagulation and provide a link between the platelet-based hemostatic system and plasmatic coagulation in vivo. Patients with Glanzman's disease [14] or Scott Syndrome [15] have reduced counts of PMP leading to bleeding disorders. On the other hand, patients with elevated levels of PMP suffer from diseases like cerebral infarction [16], acute coronary syndrome [17], sepsis [18] and venous thrombosis [19]. In the latter cases, MP from endothelial cells (EMP) are also regularly increased, reflecting injuries to the endothelial surface of the vessels. However, it is not known whether these in- creased levels of PMP and EMP are the consequence or the cause of hypercoagulation.

Within this prospective case-control study, the impact of climacteric on plasma levels of PMP and EMP in healthy women was investigated. As surrogate markers for the activation status of platelets, megakaryocytes and endothelial cells, they may play a possible role in triggering thrombotic events in postmenopausal women.

\section{Patients and Methods}

\section{Study Population and Trial Design}

In this prospective case-control study, 21 premenopausal women with or without hormone-containing OC were compared with a group of 19 menopausal women. Patients were screened and recruited from the Department of Obstetrics and Gynecology, Ludwig-Maximilians University, Klinikum Grosshadern, Munich, Germany, between November 2007 and July 2009. Women taking other medications except OC were excluded, especially postmenopausal women taking hormone replacement therapy. Participants were asked about their menstrual pattern or, accordingly, their last cycle. Premenopausal women were only enrolled in the study if they have got a normal frequent menstrual cycle between 21 and 35 days within the last year at least. The time point of blood sampling in this group was estimated during women's follicular phase between menses and ovulation. Postmenopausal women were only included in the study if their last menstrual cycle dated back 1 year or longer. Furthermore, the history of venous thromboembolism (VTE) or other disorders was evaluated and women with a history of VTE were excluded. All included participants were Caucasians and had to have normal blood counts as well as normal prothrombin time and activated partial thromboplastin time, normal fibrinogen plasma levels, and negative D-dimer at the time of investigation. To avoid other possible confounders in our study, there were further criteria which the women had to fulfill before they were enrolled: a normal body mass index between 19 and 25 (calculated after measuring height and weight), normal blood pressure $<140 / 90 \mathrm{~mm} \mathrm{Hg}$ (checked at the time point of blood sampling), non-smoking, no history of disorders and no long-time medication as well as no acute medication within the last week before blood sampling. The Human Investigation Review Board of the Ludwig-Maximilian University Munich approved the study. Signed informed consent was obtained from all women allowing analysis of all clinical and laboratory data mentioned in this paper.

\section{Blood Sampling and Isolation of MP}

Blood samples were taken by puncture of the antecubital vein without tourniquet through a 20 -gauge needle. For isolation of MP, blood samples were centrifuged at $1,550 \mathrm{~g}$ for $20 \mathrm{~min}$ within $15 \mathrm{~min}$ after sampling and stabilized in liquid nitrogen for $15 \mathrm{~min}$. Samples were stored at $-80^{\circ} \mathrm{C}$ until assayed. Samples were defrosted carefully over a period of approximately $1 \mathrm{~h}$. After centrifugation of $250 \mu \mathrm{l}$ plasma at $18,890 \mathrm{~g}$ for $30 \mathrm{~min}, 225 \mu \mathrm{l}$ of plasma/supernatant was removed. MP were resuspended after the addition of $225 \mu \mathrm{l}$ phosphate-buffered saline (PBS) containing citrate $(0.32 \%$ trisodiumcitrate) and centrifuged again for $30 \mathrm{~min}$ 
Fig. 1. Identification of MP by flow cytometry. $\mathrm{AU}=$ Arbitrary units. a MP can be found in region 1 (R1) by their dimension, as described by Nieuwland et al. [12]. SSC = Sideward scatter; FSC = forward scatter. b For negative control, MP were double stained with unspecific antibody IgG plus annexin $\mathrm{V}$ in a $\mathrm{Ca}^{2+}$-free buffer (R2). c Annexin V in $\mathrm{Ca}^{2+}$-containing buffer identifies phosphatidylserine on the MP surface with high specificity and sensitivity (R3). d Double staining of annexin $\mathrm{V}$ plus antigen (AG)-specific antibody (here: anti-CD61) determines the cell origin of MP (R4).

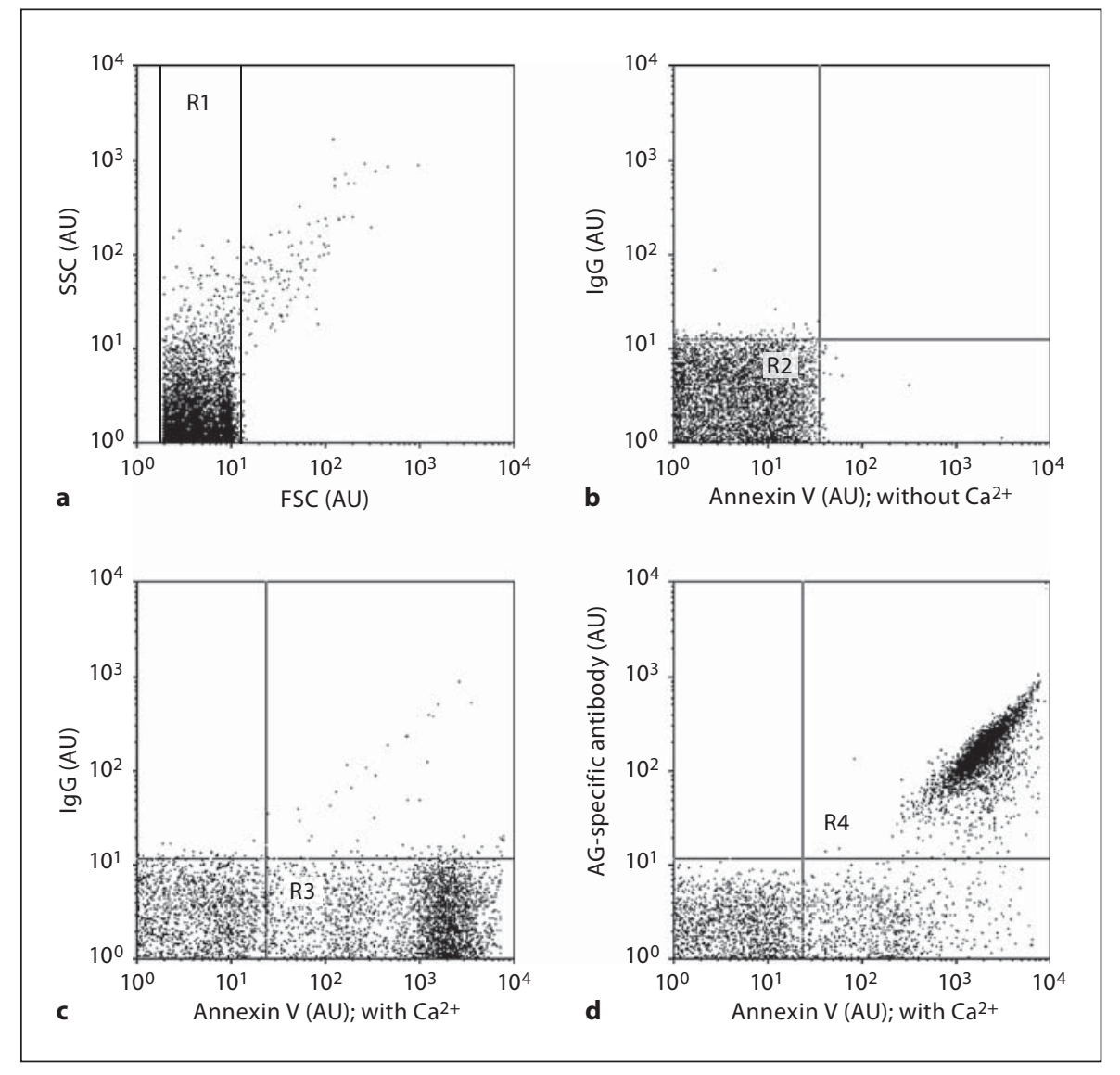

at $18,890 \mathrm{~g}$. After removal of the supernatant $(225 \mathrm{ml}), 75 \mu \mathrm{l}$ of $\mathrm{PBS} /$ citrate buffer was added, and the MP pellet was resuspended. Five microliters of the MP suspension was diluted in PBS containing $35 \mu \mathrm{l} \mathrm{CaCl} 2(2.5 \mathrm{mmol} / \mathrm{l})$ or, alternatively, $35 \mu \mathrm{l}$ citrate for annexin-V-negative controls. Finally, $5 \mu \mathrm{l}$ annexin $\mathrm{V}$ was added plus $5 \mu \mathrm{l}$ of each cell-specific monoclonal antibody or isotypematched control antibodies and incubated in the dark at room temperature for $15 \mathrm{~min}$. The reaction was stopped with $900 \mu \mathrm{l}$ of PBS containing calcium $(2.5 \mathrm{mmol} / \mathrm{l})$.

\section{Reagents}

EMP were identified via double staining with APC-labeled annexin V (Immuno Quality Products, Groningen, The Netherlands) and a fluorescein isothiocyanate (FITC)-labeled antiCD144 antibody (Acris, Hiddenhausen, Germany). PMP were detectedviaallophycocyanin(APC)-labeledannexin V,FITC-labeled anti-CD61 antibody (Immuno Quality Products), and antiCD62P antibody (Immunotech, Marseille, France) or anti-CD63 antibody (Immunotech) as a platelet activation marker. IgG-FITC and IgG-phycoerythrin (IgG-PE), both from BD Biosciences (Heidelberg, Germany), were used as antibody negative controls. All antibodies and annexin V were diluted with PBS (154 mmol/l $\mathrm{NaCl}, 1.4 \mathrm{mmol} / \mathrm{l}$ phosphate, $\mathrm{pH} 7.4$ ). Final dilutions were: annexin-V-APC 1:300 (v/v), anti-CD144-FITC 1:20, anti-CD61-FITC 1:100, anti-CD62P-PE 1:100, anti-CD63-PE 1:20, anti-IgG-FITC $1: 100$, and anti-IgG-PE 1:100.

MP Changes during Climacteric

\section{Analysis of Microparticle}

The samples were analyzed in a FACScan flow cytometer (BD Biosciences, Heidelberg) running Cell Quest Software (BD Biosciences, San Jose, Calif., USA) which was calibrated daily using beads from BD Biosciences (Heidelberg). FACScan was run on high pressure. Forward scatter, sideward scatter and fluorescence channels were set at logarithmic gain. All samples were analyzed for $1 \mathrm{~min}$. The volume analyzed in $1 \mathrm{~min}(\mathrm{~V}$; approximately $50-80$ $\mu \mathrm{l}$ ) was measured daily before analysis by taking the weight of a sample (aqua dest) before and after analysis. The gating strategy is described in figure 1 . The number of $\mathrm{MP} / \mu \mathrm{l}$ of plasma was determined by using the following formula: $\mathrm{MP} / \mathrm{l}=\mathrm{n} \times(100 \mu \mathrm{l} /$ $5 \mu \mathrm{l}) \times(950 \mu \mathrm{l} / \mathrm{V}) \times(106 / 250 \mu \mathrm{l})$ according to Berckmans et al. [20], where $\mathrm{n}=$ absolute number of MP determined by FACS analysis; $100 \mu \mathrm{l}=$ total volume of washed microparticle suspension; $5 \mu \mathrm{l}=$ pellet used for analysis; $950 \mu \mathrm{l}=$ total volume before analysis (pellet + antibodies + buffer); $250 \mu \mathrm{l}=$ original volume of the sample before isolation of MP.

\section{Statistical Methods}

Results are given as median values with interquartile ranges, if not otherwise indicated. Differences between the groups were analyzed by the non-parametric Mann-Whitney test. All statistical tests were two-tailed. $\mathrm{p}$ values $<0.05$ were regarded as statistically significant. Data were analyzed with SPSS for Windows, release 18.0. 
Table 1. MP in pre- and postmenopausal women

\begin{tabular}{|c|c|c|c|c|c|}
\hline & Total MP & $\mathrm{CD} 61+\mathrm{MP}$ & $\mathrm{CD} 62 \mathrm{P}+\mathrm{PMP}$ & CD63+ PMP & EMP \\
\hline Premenopausal women $(\mathrm{n}=21)$ & $6,159(4,427-15,023)$ & $5,364(4,384-17,167)^{*}$ & $288(139-462)^{*}$ & $281(182-551)^{*}$ & $347(207-499)$ \\
\hline Without OC $(\mathrm{n}=15)$ & $6,159(4,325-13,577)$ & $5,364(4,223-15,916)^{*}$ & $209(108-455)$ & $276(140-535)^{*}$ & $376(265-539)$ \\
\hline With OC $(n=6)$ & $7,168(4,045-16,730)$ & $7,303(4,096-18,465)$ & $323(178-536)$ & $314(250-658)^{*}$ & $289(173-502)$ \\
\hline Postmenopausal women $(\mathrm{n}=19)$ & $6,484(3,639-8,419)$ & $3,808(2,009-8,850)$ & $121(74-284)$ & $137(64-432)$ & $495(198-711)$ \\
\hline
\end{tabular}

Data are median MP $\times 10^{6} / 1$, with interquartile ranges in parentheses. ${ }^{*} \mathrm{p}<0.05$ compared to postmenopausal women.

\section{Results}

\section{Study Population}

The median age of the 21 premenopausal women was 27 years (Q25: 23 years, Q75: 35 years, range 18-40). All had regular menstrual cycles. Fifteen women had not been taking OC or other hormone-containing contraceptives for at least 1 year prior to the time of analysis. Six premenopausal women were taking OC containing 0.03 mg ethinylestradiol and $2 \mathrm{mg}$ dienogest $\left(\right.$ Valette $\left.^{\circledR}\right)$. Nineteen women were postmenopausal for at least 1 year with a median age of 61 years (Q25: 56 years, Q75: 66 years, range 53-70). None of the pre- and postmenopausal women had a history of VTE or any other diseases.

\section{Microparticle Analysis}

Counts of total annexin-V-positive MP did not differ between pre- and postmenopausal women. Similarly, no differences were measured in the numbers of CD144-exposing endothelial cell-derived MP (table 1). In contrast, the value of platelet-derived CD61-exposing MP was significantly higher in the premenopausal group $(\mathrm{p}=0.02)$ compared to postmenopausal women (fig. 2). This difference was also significant for the subgroup of premenopausal women without contraception $(\mathrm{p}=0.047)$. The highest values were measured in premenopausal women taking OC but without statistical significance because of the small number of women investigated. The plasma levels of PMP exposing the activation markers P-selectin and CD63 were also increased in women before the menopause ( $\mathrm{p}=0.024$ and 0.015 , respectively). However, the percentage fraction of PMP-exposing activation markers was similar in all investigated (sub-)groups: a median value of $4.1 \%$ was observed in premenopausal women $(4.1 \%$ without OC, $3.7 \%$ with OC) versus $4.1 \%$ in postmenopausal women (CD62P+ PMP), along with 4.3\% CD63+ PMP in premenopausal women $(4.3 \%$ without OC and $4.3 \%$ with OC vs. $4.9 \%$ in postmenopausal women).

\section{Discussion}

Our data demonstrate lower plasma levels of CD61exposing MP in postmenopausal women. These results may seem surprising as PMP are prothrombogenic and potent stimulators of coagulation. Therefore, higher CD61+ MP in postmenopausal women would be expected, possibly explaining the increased risk of thrombotic events in this population. Indeed, patients suffering from acute deep venous thrombosis or pulmonary embolism showed elevated counts of PMP [21, 22]. Ramacciotti et al. [23] could even show a positive correlation between thrombus weight and level of PMP in a mouse model. However, the elevated PMP levels reported in these other studies were present in patients with manifest thrombotic events.

Up to now, platelets were thought to be the major cell source of CD61+ MP. Accordingly, circulating CD61+ MP are commonly called 'platelet-derived microparticles' in the literature. Nevertheless, more than 10 years ago, Cramer et al. [24] showed via electronic microscopy in cell cultures that megakaryocytes constrict not only platelets but also CD61+ MP simultaneously. As megakaryocytes eject platelets directly into the blood through cytoplasmic processes penetrating the sinus wall into the sinus lumen [25], this may also result in the concurrent release of megakaryocyte-derived MP into the circulation. Although the regulation of megakaryopoiesis is not yet exactly understood, the female steroid hormone estrogen has been identified as an endogenous activator of differentiation, proliferation and proplatelet formation as well as of the constriction of megakaryocytic MP. The estrogen receptor is expressed on megakaryocytes [26]. Estradiol and progesterone are synthesized in male as well as female megakaryocytes indicating an autocrine stimulation loop of megakaryopoiesis [27]. Furthermore, addition of extracellular estradiol enhances proplatelet formation in a dose-dependent manner and an estrogen 
receptor antagonist inhibits platelet production in vivo. This would explain the higher levels of CD61-exposing MP in premenopausal women compared to those detected in postmenopausal women who are known to have no relevant estrogen level any more. In line with this conclusion, we found the highest counts of CD61-exposing MP in premenopausal women who took estrogen-containing contraceptives, even though the number of investigated women is small and limits the validity of our data. But the idea of a relevant influence of female hormones on the plasma level of CD61+ MP is supported by recent published data from our group: this subtype of plasma MP is higher in postmenopausal women with hormone replacement therapy compared to those without hormone replacement therapy [28]. The general relevance of estrogens becomes evident by the fact that women have higher mean counts of platelets as well as of CD61-exposing MP - probably mainly derived from megakaryocytes than men [29].

Unfortunately, it is not possible to distinguish MP released from platelets and megakaryocytes using common detecting antigens like fibrinogen (CD41/CD61), or von Willebrand receptor (CD42a and CD42b), as they are expressed on the surface of both cell types. Recently, however, by measuring full-length filament A and, accordingly, lysosome-associated membrane glycoprotein 1 in CD41/CD42b-exposing MP, it was shown that indeed 'PMP' without activation markers originate largely from megakaryocytes in mice [30]. Data from our own investigations indicate that this is also true in humans: killing megakaryocytes by total body irradiation in patients undergoing stem cell transplantation leads to a faster decline in CD61-exposing MP than in platelets [31]. Thus, the main population of CD61+ MP seems to be directly derived from megakaryocytes and only a small fraction, around 3-5\% in healthy humans [32], are released from platelets after cell activation. This fraction of 'real PMP' is released from activated platelets because of various reasons like high shear stress around a vessel stenosis $[33,34]$ in patients with myocardial infarction, intermittent claudicatio, or ischemic events $[35,36]$. In our study population without a history of such disorders, the percentage of platelet activation marker-positive MP in pre- and postmenopausal women was comparable with data from the literature.

We also investigated EMP levels in pre- and postmenopausal healthy women. As a specific and general marker for MP derived from vascular endothelium we used vascular endothelium-cadherin (CD144) which is thought to play an important role in endothelial cell biol-

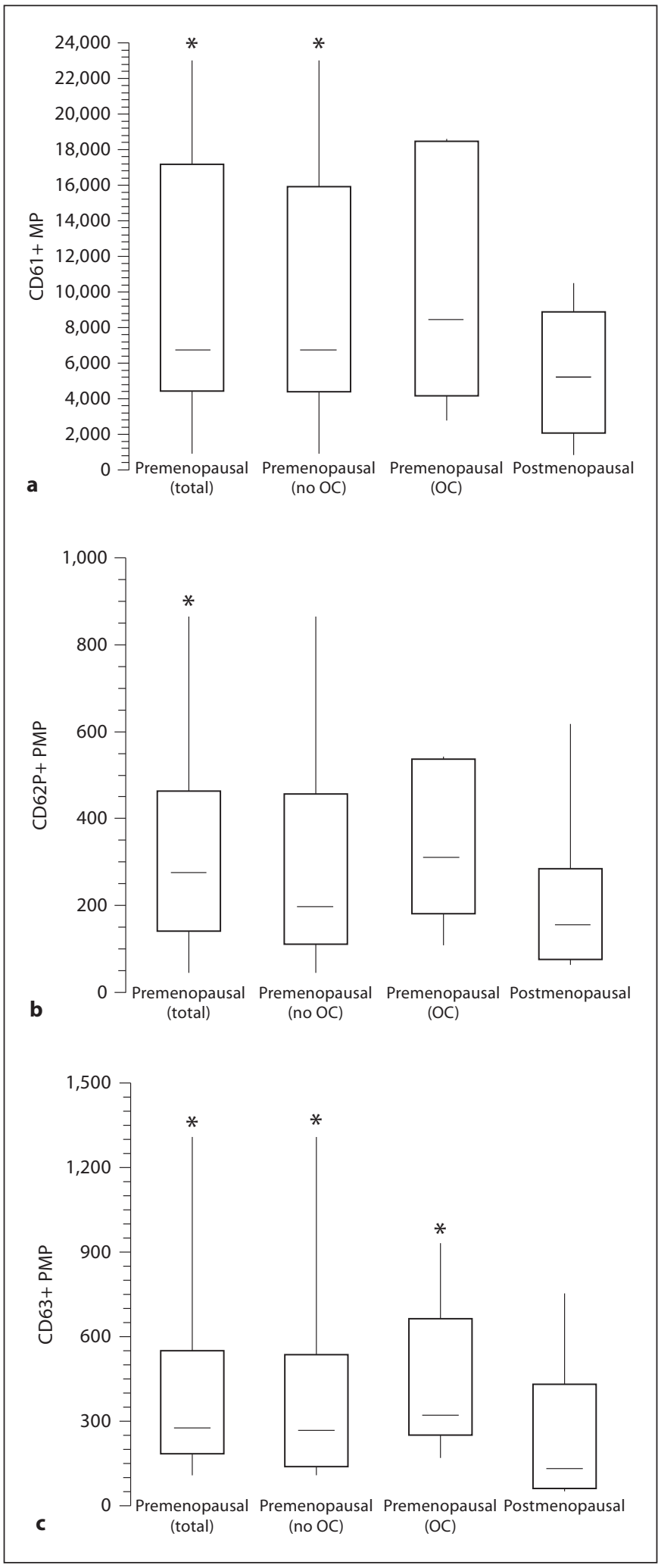

Fig. 2. Platelet-derived MP exposing CD61 (a), CD62P (b) and CD63 (c) $\left(\times 10^{6} / 1\right.$; data presented as the median and box plot). ${ }^{*} \mathrm{p}<0.05$ compared to postmenopausal women. 
ogy by controlling the cohesion and organization of the intercellular junctions [37]. Endothelium injury raises the level of circulating, CD144-exposing EMP as shown in patients with atherosclerosis and especially in those with plaque instability $[38,39]$. We did not find different levels of CD144 positivity in any investigated (sub-)group. This indicates vessel wall integrity without any damage or activation of endothelial cells within our study population.

Summing up, postmenopausal women who are known to be at high risk for vascular events showed lower PMP, probably because of female hormone deficiency. Furthermore, climacteric does not seem to have an influence on the number of EMP in healthy women. From a clinical point of view, our data show that neither PMP nor EMP are responsible for enhancing the risk of venous or arterial thromboembolism in menopausal women. Our findings are the first published impression of the impact of the climacteric on MP serum levels, but a higher number of participants is necessary to confirm our results. Fur- thermore, in a next step, we will also go more into detail of qualitative MP changes, e.g., the frequency of tissue factor-bearing MP.

\section{Acknowledgements}

The authors thank Marianne Vogel and Andrea Peichl for their technical assistance. We are very grateful to Kathleen Hiller for her careful review and editing of the manuscript as a native speaker from Iowa, USA.

'FöFoLe' of the Ludwig-Maximilian University funded Bettina Toth and her study team for her research investigation 'Plasma levels of circulating MP in postmenopausal women under hormone replacement therapy'. Fund amounts of EUR 7,500 were given for this project.

\section{Disclosure Statement}

The authors state no conflict of interest.

\section{References}

1 Rabe T, Nitsche DC, Runnebaum B: The effects of monophasic and triphasic oral contraceptives on ovarian function and endometrial thickness. Eur J Contracept Reprod Health Care 1997;2:39-51.

-2 Dratva J, Gómez Real F, Schindler C, Ackermann-Liebrich U, Gerbase MW, ProbstHensch NM, Svanes C, Omenaas ER, Neukirch F, Wjst M, Morabia A, Jarvis D, Leynaert $B$, Zemp E: Is age at menopause increasing across Europe? Results on age at menopause and determinants from two population-based studies. Menopause 2009;16: 385-394.

-3 Faddy MJ, Gosden RG, Gougeon A, Richardson SJ, Nelson JF: Accelerated disappearance of ovarian follicles in mid-life: implications for forecasting menopause. Hum Reprod 1992; 7:1342-1346.

4 Eberhardt VMS: Health, United States. Hyattsville, National Center for Health Statistics, 2001, pp 189-192.

5 Giansante C, Fiotti N, Cattin L, Da Col PG, Calabrese S: Fibrinogen, D-dimer and thrombin-antithrombin complexes in a random population sample: relationships with other cardiovascular risk factors. Thromb Haemost 1994;71:581-586.

6 Sagripanti A, Carpi A: Natural anticoagulants, aging, and thromboembolism. Exp Gerontol 1998;33:891-896.

7 Berckmans RJ, Neiuwland R, Boing AN, Romijn FP, Hack CE, Sturk A: Cell-derived microparticles circulate in healthy humans and support low grade thrombin generation. Thromb Haemost 2001;85:639-646.
8 Horstman LL, Ahn YS: Platelet microparticles: a wide-angle perspective. Crit Rev Oncol Hematol 1999;30:111-142.

-9 Biro E, Akkerman JW, Hoek FJ, Gorter G, Pronk LM, Sturk A, Nieuwland R: The phospholipid composition and cholesterol content of platelet-derived microparticles: a comparison with platelet membrane fractions. J Thromb Haemost 2005;3:2754-2763.

10 Nomura S, Tandon NN, Nakamura T, Cone J, Fukuhara S, Kambayashi J: High-shearstress-induced activation of platelets and microparticles enhances expression of cell adhesion molecules in THP-1 and endothelial cells. Atherosclerosis 2001;158:277-287.

11 Nieuwland R, Berckmans RJ, Rotteveel-Eijkman RC, Maquelin KN, Roozendaal KJ, Jansen PG, ten Have K, Eijsman L, Hack CE, Sturk A: Cell-derived microparticles generated in patients during cardiopulmonary bypass are highly procoagulant. Circulation 1997;96:3534-3541.

12 Thiagarajan P, Tait JF: Collagen-induced exposure of anionic phospholipid in platelets and platelet-derived microparticles. J Biol Chem 1991;266:24302-24307.

13 Gilbert GE, Sims PJ, Wiedmer T, Furie B, Furie BC, Shattil SJ: Platelet-derived microparticles express high affinity receptors for factor VIII. J Biol Chem 1991;266:17261-17268.

14 Gemmell CH, Sefton MV, Yeo EL: Plateletderived microparticle formation involves glycoprotein IIb-IIIa. Inhibition by RGDS and a Glanzmann's thrombasthenia defect. J Biol Chem 1993;268:14586-14589.
15 Sims PJ, Wiedmer T, Esmon CT, Weiss HJ, Shattil SJ: Assembly of the platelet prothrombinase complex is linked to vesiculation of the platelet plasma membrane. Studies in Scott syndrome: an isolated defect in platelet procoagulant activity. J Biol Chem 1989;264: 17049-17057.

16 Kuriyama N, Nagakane Y, Hosomi A, Ohara T, Kasai T, Harada S, Takeda K, Yamada K, Ozasa K, Tokuda T, Watanabe Y, Mizuno T, Nakagawa M: Evaluation of factors associated with elevated levels of platelet-derived microparticles in the acute phase of cerebral infarction. Clin Appl Thromb Hemost 2010; $16: 26-32$.

17 Li X, Cong H: Platelet-derived microparticles and the potential of glycoprotein IIb/IIIa antagonists in treating acute coronary syndrome.Tex Heart Inst J 2009;36:134-139.

18 Joop K, Berckmans RJ, Nieuwland R, Berkhout J, Romijn FP, Hack CE, Sturk A: Microparticles from patients with multiple organ dysfunction syndrome and sepsis support coagulation through multiple mechanisms. Thromb Haemost 2001;85:810-820.

19 Myers DD, Hawley AE, Farris DM, Wrobleski SK, Thanaporn P, Schaub RG, Wagner DD, Kumar A, Wakefield TW: P-selectin and leukocyte microparticles are associated with venous thrombogenesis. J Vasc Surg 2003;38: 1075-1089.

20 Berckmans RJ, Nieuwland R, Böing AN, Romijn FP, Hack CE, Sturk A: Cell-derived microparticles circulate in healthy humans and support low grade thrombin generation. Thromb Haemost 2001;83:639-646. 
21 Bal L, Ederhy S, Di Angelantonio E, Toti F, Zobairi F, Dufaitre G, Meuleman C, Mallat Z, Boccara F, Tedgui A, Freyssinet JM, Cohen A: Factors influencing the level of circulating procoagulant microparticles in acute pulmonary embolism. Arch Cardiovasc Dis 2010;103:394-403.

22 Rectenwald JE, Myers DD Jr, Hawley AE, Longo C, Henke PK, Guire KE, Schmaier $\mathrm{AH}$, Wakefield TW: D-dimer, P-selectin, and microparticles: novel markers to predict deep venous thrombosis. A pilot study. Thromb Haemost 2005;94:1312-1317.

-23 Ramacciotti E, Hawley AE, Farris DM, Ballard NE, Wrobleski SK, Myers DD Jr, Henke PK, Wakefield TW: Leukocyte- and plateletderived microparticles correlate with thrombus weight and tissue factor activity in an experimental mouse model of venous thrombosis. Thromb Haemost 2009;101:748-754.

- 24 Cramer EM, Norol F, Guichard J, BretonGorius J, Vainchenker W, Massé JM, Debili $\mathrm{N}$ : Ultrastructure of platelet formation by human megakaryocytes cultured with the Mpl ligand. Blood 1997;89:2336-2346.

-25 Travlos GS: Normal structure, function, and histology of the bone marrow. Toxicol Pathol 2006;34:548-565.

26 Tarantino MD, Kunicki TJ, Nugent DJ: The estrogen receptor is present in human megakaryocytes. Ann NY Acad Sci 1994;714:293296.

-27 Nagata Y, Yoshikawa J, Hashimoto A, Yamamoto M, Payne AH, Todokoro K: Proplatelet formation of megakaryocytes is triggered by autocrine-synthesized estradiol. Genes Dev 2003;17:2864-2869.
28 Rank A, Nieuwland R, Nikolajek K, Rösner S, Wallwiener LM, Hiller E, Toth B: Hormone replacement therapy leads to increased plasma levels of platelet derived microparticles in postmenopausal women. Arch Gynecol Obstet 2012;285:1035-1041.

29 Stevens RF, Alexander MK: A sex difference in the platelet count. Br J Haematol 1977;37: 295-300.

30 Flaumenhaft R, Dilks JR, Richardson J, Alden E, Patel-Hett SR, Battinelli E, Klement GL, Sola-Visner M, Italiano JE Jr: Megakaryocyte-derived microparticles: direct visualization and distinction from platelet-derived microparticles. Blood 2009;113:11121121.

31 Rank A, Nieuwland R, Delker R, Köhler A, Toth B, Pihusch V, Wilkowski R, Pihusch R: Cellular origin of platelet-derived microparticles in vivo. Thromb Res 2010;126:e255e259.

32 Toth B, Nikolajek K, Rank A, Nieuwland R, Lohse P, Pihusch V, Friese K, Thaler CJ: Gender-specific and menstrual cycle dependent differences in circulating microparticles. Platelets 2007;18:515-521.

33 Warren BA, Vales O: The release of vesicles from platelets following adhesion to vessel walls in vitro. Br J Exp Pathol 1972;53:206215.

34 Miyazaki Y, Nomura S, Miyake T, Kagawa H, Kitada C, Taniguchi H, Komiyama Y, Fujimura Y, Ikeda Y, Fukuhara S: High shear stress can initiate both platelet aggregation and shedding of procoagulant containing microparticles. Blood 1996;88: 3456-3464.
5 van der Zee PM, Biró E, Ko Y, de Winter RJ, Hack CE, Sturk A, Nieuwland R: P-selectinand CD63-exposing platelet microparticles reflect platelet activation in peripheral arterial disease and myocardial infarction. Clin Chem 2006;52:657-664.

36 Tan KT, Tayebjee MH, Lynd C, Blann AD, Lip GY: Platelet microparticles and soluble P selectin in peripheral artery disease: relationship to extent of disease and platelet activation markers. Ann Med 2005;37:61-66.

37 Corada M, Liao F, Lindgren M, Lampugnani MG, Breviario F, Frank R, Muller WA, Hicklin DJ, Bohlen P, Dejana E: Monoclonal antibodies directed to different regions of vascular endothelial cadherin extracellular domain affect adhesion and clustering of the protein and modulate endothelial permeability. Blood 2001;97:1679-1684.

38 Simak J, Holada K, Risitano AM, Zivny JH, Young NS, Vostal JG: Elevated circulating endothelial membrane microparticles in paroxysmal nocturnal haemoglobinuria. Brit J Haematol 2004;125: 804-813.

39 Koga H, Sugiyama S, Kugiyama K, Watanabe K, Fukushima H, Tanaka T, Sakamoto T, Yoshimura M, Jinnouchi $\mathrm{H}$, Ogawa $\mathrm{H}$ : Elevated levels of VE-cadherin-positive endothelial microparticles in patients with type 2 diabetes mellitus and coronary artery disease. $J$ Am Coll Cardiol 2005;45:1622-1630. 